\title{
Coagulation effects of mannitol in combination with $0.9 \%$ normal saline or hydroxyethyl starch in patients undergoing supratentorial craniotomy: A preliminary report
}

\author{
Hemanshu Prabhakar, Gyaninder Pal Singh, Parmod Kumar Bithal, Mani Kalaivani
}

\begin{abstract}
Background: Neurosurgical patients often require administration of both, mannitol and hydroxyethyl starch (HES). A recent in vitro study demonstrated that HES in combination with mannitol could disturb coagulation parameters and should be avoided in neurosurgical practice. The aim of our study was to evaluate coagulation abnormalities due to mannitol when administered alone and in combination with HES in patients undergoing craniotomy for various intracranial brain tumours. Materials and Methods: We enrolled 30 adult patients undergoing craniotomy. Patients were randomised into two groups using a computer generated randomisation chart. Interventions: Group A: Patients received $10 \mathrm{ml} / \mathrm{kg} 0.9 \%$ normal saline and I g/kg mannitol and Group B: Patients received $10 \mathrm{ml} / \mathrm{kg}$, HES 130/0.4, and I gm/kg mannitol; immediately after induction of general anaesthesia. Rotational thromboelastography was done immediately after induction of general anaesthesia and $5 \mathrm{~min}$ after administration of mannitol. Measured parameters of blood coagulation were clotting time (CT) and clot formation time (CFT) with EXTEM and maximum clot firmness (MCF) with EXTEM and (FIBTEM). Results: Fourteen patients in each group completed the study. Insignificant change was noted in CT; CFT altered significantly from the baseline in both the groups $(P<0.05)$. MCF with FIBTEM did not change significantly from baseline $(P>0.05)$, but significantly differed between groups $(P=0.00 \mathrm{I})$. However, all values were in normal range. Conclusion: Mannitol I g/ $/ \mathrm{kg}$ and HES $10 \mathrm{ml} / \mathrm{kg}$ can be safely administered in patients undergoing craniotomy for supratentorial tumours, without clinically significant changes in coagulation parameters.
\end{abstract}

Key words: Coagulation, colloid, mannitol, neuroanaesthesia, thromboelastography

\section{INTRODUCTION}

The perioperative assessment of coagulation profile in neurosurgical patients has shown to produce a hypercoagulable state. ${ }^{[1,2]}$ Fluids such as mannitol and

\begin{tabular}{|l|l|}
\hline \multicolumn{2}{|c|}{ Access this article online } \\
\hline Quick Response Code: & Website: \\
\hline & www.jnaccjournal.org \\
\cline { 2 - 2 } & \\
\hline
\end{tabular}

colloids are routinely used in neurosurgical practice. Colloids are used to replace the intravascular volume, whereas mannitol is used to reduce cerebral oedema and intracranial pressure. In vitro and in vivo studies have shown that both crystalloids and colloids induce a hypercoagulable state. ${ }^{[3-5]}$ There is a demonstrable effect of mannitol administration on blood viscosity ${ }^{[6]}$ and osmolality. ${ }^{[7]}$ However, the effect of mannitol on blood coagulation is unknown. In an experimental in vitro model, Lindroos and colleagues ${ }^{[8]}$ assessed the possible effects of mannitol on whole blood coagulation and determined whether Ringer acetate or hydroxyethyl starch (HES) $130 / 0.4$ had any additional effects. The authors concluded that HES, in combination with mannitol,

Departments of Neuroanaesthesiology, ${ }^{1}$ Biostatistics, Neurosciences Centre, All India Institute of Medical Sciences, New Delhi, India 
could disturb homeostasis and should be avoided in neurosurgical practice. ${ }^{[8]}$ As neurosurgical patients undergoing craniotomy often require administration of both, mannitol and HES, we decided to evaluate the effects of the two fluids when given in combination. The aim of this prospective, randomised, experimental study was to evaluate coagulation abnormalities (as detected by thromboelastography (TEG)) due to mannitol when administered alone and in combination with HES in patients undergoing craniotomy for various intracranial brain tumours.

\section{MATERIALS AND METHODS}

After approval from ethics committee and informed consent from patient or their close relatives, we enrolled 30 adult patients scheduled to undergo craniotomy for supratentorial tumour. Patients with history of coagulopathies, those on anticoagulant and nonsteroidal anti-inflammatory analgesic drugs, paediatric patients, history of intracranial surgery and trauma and non-consenting patients were excluded from the study. Demographic data such as age, gender, weight, diagnosis and personal history specific for smoking was noted. Based on a computer generated randomisation chart, patients were grouped in two: Group I: Patients received $10 \mathrm{ml} / \mathrm{kg} 0.9 \%$ normal saline and $1 \mathrm{gm} / \mathrm{kg}$ mannitol, immediately after induction of general anaesthesia.

Group II: Patients received $10 \mathrm{ml} / \mathrm{kg}$ HES 130/0.4 and $1 \mathrm{~g} / \mathrm{kg}$ mannitol, immediately after induction of general anaesthesia.

All patients were premedicated with intramuscular glycopyrrolate $0.2 \mathrm{mg} 1 \mathrm{~h}$ prior to surgery. Mannitol, in both groups, was administered over a period of $20 \mathrm{~min}$.

A standard anaesthetic technique was followed for all patients. An $18 \mathrm{G}$ venous cannula was inserted on the dorsum of hand and normal saline or HES infusion was started slowly. Anaesthesia was induced with fentanyl $2 \mu \mathrm{g} / \mathrm{kg}$ and thiopentone $4-5 \mathrm{mg} / \mathrm{kg}$. Tracheal intubation was performed after rocuronium $1 \mathrm{mg} / \mathrm{kg}$. Anaesthesia was maintained using isoflurane in oxygen and nitrous oxide mixture (1:2). Mechanical ventilation will be facilitated with intermittent boluses of fentanyl $1 \mu \mathrm{g} / \mathrm{kg}$ (whenever heart rate and mean blood pressure exceed $20 \%$ of baseline) and vecuronium $0.1 \mathrm{mg} / \mathrm{kg}$, to target end tidal carbon dioxide of $32 \pm 2 \mathrm{mmHg}$. Arterial blood samples were taken for gas analysis and rotational thromboelastography (ROTEM) at two time intervals.

1. A baseline sample, taken immediately after induction of general anaesthesia (Sample a)

2. Five minutes after administration of mannitol (Sample b).

Further management of the patient was at the discretion of the attending anaesthesiologists and per the routine protocols of our department. Intraoperative fluid administration consisted primarily of $0.9 \%$ normal saline and blood and blood products were given if required, depending on intraoperative blood loss. Any intraoperative event relating to coagulation system, such as excessive bleeding from surgical site was carefully monitored. Postoperatively, all patients were shifted to the neurosurgical intensive care unit (ICU) for supportive care and further management. ROTEM was carried out using ROTEM ${ }^{\circledast}$ Pentapharm $\mathrm{GmbH}$, Munich, Germany. The coagulation was allowed to proceed for $30 \mathrm{~min}$ and assays performed were EXTEM and FIBTEM in the manner described by the manufacturers. Automatically measured parameters of blood coagulation were clotting time (CT) and clot formation time (CFT) with EXTEM and maximum clot firmness (MCF) with EXTEM and FIBTEM.

Statistical analysis was done using software STATA 9.1 (College Station, Texas, USA). Data are presented as mean (standard deviation (SD)), number or percentage. Shapiro-Wilk test was used to assess the normality of the data. Levene's test was applied to compare the difference in standard deviations before applying Student's $t$-test for independent samples. The difference in means between the groups was compared using two sample $t$-test with equal/unequal variance as applicable and the change in mean values from baseline within each group was done using the paired $t$-test. Baseline imbalance between the two groups was corrected using analysis of covariance (ANCOVA). The value of $P$ less than 0.05 was considered statistically significant.

\section{RESULTS}

Thirty patients were enrolled for the study. Data of 28 patients was used for final analysis as one patient in each group was excluded due to technical failure in ROTEM and results could not be obtained. Demographic data and baseline haemoglobin $(\mathrm{Hb})$ and haematocrit (Hct) values were comparable [Table 1]. A total of four patients (three patients in group I and one patient in group II) gave history of smoking. Insignificant change was noted in CT after dilution with study fluids and the values were also comparable between the groups [Table 2]. However, the CFT altered significantly from the baseline in both the groups and the change was significantly more in group II. MCF with EXTEM did not change significantly from baseline values, but significant difference was noted between groups [Table 2]. MCF with FIBTEM did not change significantly from baseline value but differed significantly between groups [Table 3]. Significant change was also noted in $\mathrm{Hb}$ and Hct values after dilution with fluids in both the groups; however the two groups were comparable [Table 3]. No complications were noted in either group. 


\section{DISCUSSION}

ROTEM is a validated method to detect alterations in coagulation system and a useful technique for evaluating hypercoagulability. Coagulation abnormalities can be easily detected using ROTEM, which may not be detected by routine laboratory tests. The usefulness of ROTEM has already been shown in cardiac surgery and liver transplants. There is limited literature of its use in neurosurgical practice, but evident enough to justify its importance. ${ }^{[1,2,9,10]}$ Studies have shown that decrease in MCF as detected by ROTEM, is associated with increased postoperative blood loss. ${ }^{[5]}$ ROTEM also helps in guiding administration of various blood products such as fresh

\section{Table 1: Demographic profile and baseline} characteristics [Mean (SD)] or number

\begin{tabular}{lccc}
\hline & $\begin{array}{c}\text { Group I } \\
(\boldsymbol{n}=\mathbf{1 4})\end{array}$ & $\begin{array}{c}\text { Group } \\
\text { II }(\boldsymbol{n}=\mathbf{1 4})\end{array}$ & P value \\
\hline Age (years) & $36.7(12.8)$ & $36.4(8.2)$ & 0.94 \\
Weight (kg) & $63.8(11.3)$ & $58.5(9.2)$ & 0.18 \\
Male: female & $10: 4$ & $5: 9$ & \\
Baseline & $13.4(1.6)$ & $13.2(1.9)$ & 0.7 \\
Hb (gm\%) & & & \\
Baseline & $40.9(5)$ & $40.4(5.6)$ & 0.12 \\
Hct (\%) & & \\
\hline$n=$ Number of patients, HB = hemoglobin, HCT = hematocrit &
\end{tabular}

frozen plasma, platelet concentrates and fibrinogen.

Our results revealed that infusion of mannitol alone or in combination with HES resulted in impaired coagulation as suggested by decreased MCF and CFT. Some of our findings are similar to those observed in an in vitro study by Lindroos and colleagues. ${ }^{[8]}$ The authors reported increased CFT and decreased MCF in all dilutions compared with control. They also found that that fibrinogen-dependent MCF in $10 \mathrm{vol} \%$ dilution of mannitol with HES was lower than MCF with Ringer acetate dilution of same concentration. In our study also, the fibrinogen-dependent MCF decreased in mannitol-HES combination when compared with normal saline-mannitol combination, and the results were statistically significant. An interesting observation was that although the changes in coagulation parameters were statistically significant, all values were within normal range. The reference values taken for each assay was as provided by the manufacturers.

There is paucity of literature on the effects of mannitol administration on blood coagulation system. Mannitol is routinely used in neurosurgical patients and the importance of clotting system in neurosurgical patients cannot be overemphasised.

Colloids, like HES, are also in routine use as intravascular replacement fluids. HES has been shown to impair clot formation and firmness in vitro. ${ }^{[4]}$ Although the exact

Table 2: Clotting time, clot formation time and maximum clot firmness with EXTEM [mean (SD)]

\begin{tabular}{lccccccccc}
\hline & $\begin{array}{c}\text { Ex CT } \\
\mathbf{a}(\mathbf{s})\end{array}$ & $\begin{array}{c}\text { Ex CT } \\
\mathbf{b}(\mathbf{s})\end{array}$ & $\boldsymbol{P}^{\dagger}$ & Ex CFT a (s) & $\begin{array}{c}\text { Ex CFT } \\
\mathbf{b}(\mathbf{s})\end{array}$ & $P^{\dagger}$ & $\begin{array}{c}\text { Ex } \\
\mathbf{M C F} \\
\mathbf{a}(\mathbf{m m})\end{array}$ & $\begin{array}{c}\text { Ex } \\
\mathbf{M C F} \\
\mathbf{b}^{\#}(\mathbf{m m})\end{array}$ & $P^{\dagger}$ \\
\hline $\begin{array}{l}\text { Group } \\
\mathrm{I}(n=14)\end{array}$ & $66.4(17.3)$ & $65.5(20.2)$ & 0.98 & $102.3(48.20)$ & $108.4(17.2)$ & $0.0001^{*}$ & $60.1(6.8)$ & $58.1(8.5)$ & 0.75 \\
$\begin{array}{l}\text { Group } \\
\text { II }(n=14)\end{array}$ & $62.3(12.2)$ & $72.6(20.2)$ & 0.21 & $91.4(20.9)$ & $141.7(17.2)$ & $0.0001^{*}$ & $59.4(5.1)$ & $51.3(8.5)$ & 0.06 \\
$\mathrm{P}^{\ddagger}$ & 0.47 & 0.37 & 0.44 & $0.001^{*}$ & $0.02^{*}$ & $0.04^{*}$ & & & \\
\hline
\end{tabular}

$n=$ number of patients, Ex = EXTEM, CT a = baseline clotting time, CT b = clotting time after infusion of study fluids, CFT a = baseline clot formation time, CFT $b=$ clot formation time after infusion of study fluids, MCF $a=$ baseline maximum clot firmness, MCF $b=$ maximum clot firmness after infusion of study fluids, $\mathrm{SD}=$ standard deviation. ${ }^{\dagger} P$ value of within group comparison, ${ }^{\dagger} P$ value between group comparison, ${ }^{*} P$ value showing significance, ${ }^{\#}$ Values adjusted to the baseline difference

Table 3: Maximum clot firmness with FIBTEM and haemoglobin and haematocrit changes after dilution [mean (SD)]

\begin{tabular}{|c|c|c|c|c|c|c|c|c|c|}
\hline & $\begin{array}{l}\text { Fib MCF } \\
\text { a (mm) }\end{array}$ & $\begin{array}{l}\text { Fib MCF } \\
\text { b (mm) }\end{array}$ & $P^{\dagger}$ & $\begin{array}{l}\mathrm{Hb} \text { a } \\
(\mathrm{g} \%)\end{array}$ & $\begin{array}{l}\mathrm{Hb} b \\
(\mathrm{~g} \%)\end{array}$ & $P^{\dagger}$ & $\begin{array}{l}\text { Hct } \\
\text { a (\%) }\end{array}$ & $\begin{array}{l}\text { Hct } \\
\text { b (\%) }\end{array}$ & $P^{\dagger}$ \\
\hline $\begin{array}{l}\text { Group } \\
\mathrm{I}(n=14)\end{array}$ & $12.3(4.4)$ & $17.5(4.8)$ & 0.13 & 13.4 (1.6) & 11.6 (1.6) & $0.0001^{*}$ & 40.9 (5) & $35.7(4.7)$ & $0.0001^{*}$ \\
\hline $\begin{array}{l}\text { Group } \\
\text { II }(n=14)\end{array}$ & 17.9 (13.7) & $10.6(4.8)$ & 0.06 & $13.2(1.9)$ & $10.6(1.6)$ & $0.0001^{*}$ & $40.4(5.6)$ & $32.9(5.1)$ & $0.0001^{*}$ \\
\hline $\mathrm{P}^{\ddagger}$ & 0.10 & & $0.001^{*}$ & 0.7 & & 0.12 & 0.8 & & 0.15 \\
\hline
\end{tabular}

$n=$ number of patients, FIB = FIBTEM, MCF a = baseline maximum clot firmness, MCF b = maximum clot firmness after infusion of study fluids, Hb a = baseline haemoglobin, $\mathrm{Hb} \mathrm{b}=$ haemoglobin after infusion of study fluids, Hct a = baseline haematocrit, Hct $b=$ haematocrit after infusion of study fluids, SD = standard deviation. ${ }^{\dagger} P$ value of with-in group comparison, ${ }^{\ddagger} P$ value between group comparison, ${ }^{*} P$ value showing significance 
mechanism for deranged coagulation caused by HES remains unclear, possible mechanisms include: Inhibition of platelet function, decrease of coagulation factors (Von Willebrand factor and factor VIII), decrease in plasma fibrinogen levels or increased fibrinolysis. ${ }^{[1]]}$ However, HES 130/0.4 is considered safest HES since a lower blood loss has been shown with use of the former compared to HES 200/0.5, in patients undergoing major surgery. ${ }^{[12]}$

Increased coagulability begins after induction of anaesthesia and continues to increase throughout surgery in patients undergoing craniotomy. ${ }^{[1,2]}$ To avoid any surgical interference, we collected samples prior to surgical incision. Our patients varied in their pathological diagnosis with tumours being epidermoids and gliomas that bleed less compared with meningiomas and vascular malformations that bleed massively at times. Therefore, their intraoperative blood losses and duration of surgery also varied, which is understandable. However, we did look for intraoperative signs of coagulopathy.

One of the limitations of our study is the small sample size. Considering CT as the primary outcome of our interest, the power estimate of our study is $16 \%$. This requires sample size of 172 patients per group, which is logistically not possible. However, considering other parameters, such as CFT and MCF, which altered significantly, our study has power of nearly $99 \%$. Our study is also limited by the fact that only single doses of mannitol and HES were studied for coagulation abnormalities. We feel that the doses used by us are standard doses, which are used popularly in clinical practice. Occasionally, in cases of excessive blood loss one may resort to using higher doses of HES $(50 \mathrm{ml} / \mathrm{kg} / 24 \mathrm{~h})$. At the same time, perioperative assessment of ROTEM should have been done, which could have given more clinically useful findings.

In conclusion, in vitro studies/conditions are completely dissimilar to in vivo environment. Interaction of drugs and fluids with vascular endothelium may be varied during surgical stress. Our study demonstrates that mannitol $1 \mathrm{~g} / \mathrm{kg}$ and HES $10 \mathrm{ml} / \mathrm{kg}$ may be safely administered in patients undergoing craniotomy for supratentorial tumours, without clinically significant changes in coagulation parameters.

\section{REFERENCES}

1. Abrahams JM, Torchia B, McGarvey M, Putt M, Baranov D, Sinson GP. Perioperative assessment of coagulability in neurosurgical patients using thromboelastography. Surg Neurol 2002;58:5-11.

2. Goobie SM, Soriano SG, Zurakowski D, McGowan FX, Rockoff MA. Hemostatic changes in pediatric neurosurgical patients as evaluated by thromboelastograph. Anesth Analg 2001;93:887-92.

3. Ekseth K, Abildgaard L, Vegfors M, Berg-Johnsen J, Engdahl O. The in vitro effects of crystalloids and colloids on coagulation. Anaesthesia 2002;57:1102-8.

4. Niemi TT, Kuitunen AH. Artificial colloids impair haemostasis. An in vitro study using thromboelastometry coagulation analysis. Acta Anaesthesiol Scand 2005;49:373-8.

5. Niemi TT, Suojaranta-Ylinen RT, Kukkonen SI, Kuitunen AH. Gelatin and hydroxyl-ethyl starch, but not albumin, impair hemostasis after cardiac surgery. Anesth Analg 2006;102:998-1006.

6. Burke AM, Quest DO, Chien S, Cerri C. The effects of mannitol on blood viscosity. J Neurosurg 1981;55:550-3.

7. Manninen PH, Lam AM, Gelb AW, Brown SC. The effect of high-dose mannitol on serum and urine electrolytes and osmolality in neurosurgical patients. Can J Anaesth 1987;34:442-6.

8. Lindroos AC, Schramko A, Tanskanen P, Niemi T. Effect of the combination of mannitol and ringer acetate or hydroxyethyl starch on whole blood coagulation in vitro. J Neurosurg Anaesthesiol 2010;22:16-20.

9. Fujii Y, Tanaka R, Takeuchi S, Koike T, Minakawa T, Sasaki O. Serial changes in hemostasis after intracranial surgery. Neurosurgery 1994;35:26-33.

10. van der Sande JJ, Veltkamp JJ, Bouwhuis-Hoogerwerf ML. Hemostasis and intracranial surgery. J Neurosurg 1983;58:693-8.

11. Sossdorf M, Marx S, Schaarschmidt B, Otto GP, Claus RA, Reinhart K, et al. HES 130/0.4 impairs haemostasis and stimulates pro-inflammatory blood platelet function. Crit Care 2009;13:R208.

12. Kozek-Langenecker SA, Jungheinrich C, Sauermann W, Van der Linden P. The effects of hydroxyethyl starch 130/0.4 (6\%) on blood loss and use of blood products in major surgery: A pooled analysis of randomized clinical trials. Anesth Analg 2008;107:382-90.

How to cite this article: Prabhakar H, Singh GP, Bithal PK, Kalaivani M. Coagulation effects of mannitol in combination with 0.9 normal saline or hydroxyethyl starch in patients undergoing supratentorial craniotomy: A preliminary report. J Neuroanaesthesiol Crit Care 2014;1:56-9.

Source of Support: Nil, Conflict of Interest: None declared. 